\title{
Ginsenoside Rh2 inhibits human A172 glioma cell proliferation and induces cell cycle arrest status via modulating Akt signaling pathway
}

\author{
KAI-FEI LI, CHUN-MIN KANG, XIAO-FENG YIN, HAI-XIA LI, \\ ZHUO-YU CHEN, YAO LI, QIONG ZHANG and YU-RONG QIU
}

Laboratory Medicine Center, Nanfang Hospital, Southern Medical University, Guangzhou, Guangdong 510515, P.R. China

Received July 12, 2017; Accepted November 13, 2017

DOI: $10.3892 / \mathrm{mmr} .2017 .8193$

\begin{abstract}
Ginsenoside Rh2 (G-Rh2), the main bioactive component in American ginseng, is known to exert a wide variety of biological activities. Accumulating evidence suggests that G-Rh2 inhibits cell proliferation and induces apoptosis of tumor cells. However, the possible mechanism through which G-Rh2 exerts its action on malignant glioma cells have not been completely elucidated. The findings of the present study demonstrated that G-Rh2 decreased the viability of glioma cells in a dose- and time-dependent manner, and induced cell cycle arrest. G-Rh2-induced cell cycle arrest was accompanied by the downregulation of cyclin-dependent kinase 4 and Cyclin E. In addition, G-Rh2 markedly reduced the expression of total- RAC- $\alpha$ serine/threonine-protein kinase (Akt) and the levels of phosphorylated-Akt. These findings provide mechanistic details of how G-Rh2 acts on glioma cells and suggest that G-Rh2 may function as a potential anti-cancer drug for glioma treatment.
\end{abstract}

\section{Introduction}

Glioma, originated from the glial cell, is the most common intracranial primary tumors, accounting for $80 \%$ of malignant brain tumors (1). Overall age-adjusted incidence rate for all gliomas ranges from 4.67 to 5.73 per 100 thousand persons with a median survival 2 to 5 years. However, the median survival is only one year for patients with glioblastoma multiforme (GBM) (2,3). Currently, invasive therapy achieves little benefit in glioma owing to their less effective penetrance to brain tissue. Adjuvant chemotherapy also has minimal efficacy. Tumor heterogeneity, low membrane permeability,

\footnotetext{
Correspondence to: Professor Yu-Rong Qiu, Laboratory Medicine Center, Nanfang Hospital, Southern Medical University, 1838 North Guangzhou Avenue, Guangzhou, Guangdong 510515, P.R. China E-mail: qiuyuronggz@126.com
}

Key words: ginsenoside Rh2, glioma, proliferation, cell cycle arrest, Akt pathway pre systemic metabolism, and blood brain barrier (BBB) contribute to therapeutic resistance and tumor recurrence (4). Therefore, novel approaches for the treatment of glioma are critically required.

Ginseng, one of the most commonly used Chinese herbal medicines in the United States, contains multiple effective components which have been reported to have a wide variety of biological activities including immunomodulatory, neuroprotection, anti-aging and anti-tumor effects (5-8). Ginsenoside Rh2 (G-Rh2), a dammarane-type glycoside compounds (Fig. 1A) separated from Ginseng has been reported to induce apoptosis by reactive oxygen species or activating the p53 pathway in several tumors (9-11). In recent years, increasing insights into the mechanism of G-Rh2 actions have been elucidated. G-Rh2 induced internalization of rafts and caveolae, have reported to inactivate Akt and induce cell apoptosis (12). In addition, G-Rh2 has been shown to affect cell viability by modulating autophagy (13). These studies suggest that anti-tumor effect mediated by G-Rh2 might involve multiply mechanisms.

Cell cycle deregulation is one of the most frequent alterations associated with tumor development. Thus, the blockade of cell cycle is regarded as a feasible strategy for targeting tumor growth. In the process of cell proliferation, the conversion of G1-S phase is considered to be the key step (14). Upstream signaling molecules such as hormones or cytokines causes cell proliferation or inhibition, which ultimately affects retinoblastoma protein $(\mathrm{pRb})$. When phosphorylated by the $\mathrm{CDKs}, \mathrm{pRb}$ separate from the E2F factors to relieve its inhibitory effect. Therefore, E2F factors promote the transcriptions of genes causing cells to enter the $S$ phase from the $G 1$ phase $(15,16)$. CDKs activity is regulated by exact regulatory networks, including the activated factor: Mainly CyclinD and CyclinE, and the inhibitory factor: INK4 and CIP/KIP families. Intensive research about the characteristics of tumor cell cycle progression is helpful to select the optimal target drugs (17).

Previous evidence demonstrates that G-Rh2 blocks the tumor cell proliferation via multiple mechanisms (9-13). G-Rh2 induced the cell differentiation and cycle arrest in human leukemia by up-regulating TGF- $\beta$ expression (18). G-Rh2 decreased the cells proliferation and induced the cell apoptosis through inhibiting EGFR expression in gliomas (19). However, 
the underlying molecular mechanistic details of G-Rh2 effects on glioma malignant cells have not been completely elucidated. In this study, we have investigated the effects of G-Rh2 on proliferation, cell cycle regulation of human glioma cells and the underlying molecular mechanisms involved.

\section{Materials and methods}

Cell culture. The glioma cell lines A172 and U87MG were purchased from the American Type Culture Collection (ATCC, Manassas, VA, USA). Cells were cultured in Dulbecco's modified Eagle's medium (DMEM; Gibco, Thermo Fisher Scientific Inc., Grand Island, NY, USA) supplemented with $10 \%$ fetal bovine serum (FBS; Biowest, Nuaillé, France), $100 \mathrm{U} / \mathrm{ml}$ penicillin and $0.1 \mathrm{mg} / \mathrm{ml}$ streptomycin and maintained in a humidified incubator at $37^{\circ} \mathrm{C}$ with $5 \% \mathrm{CO}_{2}$.

Reagents. G-Rh2 (purity $\geq 98 \%$ ) used for the present study was purchased from Aladdin (Shanghai, China). The identity was confirmed by liquid chromatography-mass spectrometry. G-Rh2 was prepared in DMSO (Sigma-Aldrich, St. Louis, MO, USA) at a stock concentration of $10 \mathrm{mM}$ and diluted with fresh complete medium immediately before use. Cell Counting Kit-8 (CCK-8; Dojindo Laboratories, Tokyo, Japan), Cell apoptosis kit and Cell Cycle kit (KeyGEN BioTECH, Jiangsu, China) were obtained from commercial sources. Antibodies: CDK4 (cat. no. A0336), CyclinD (cat. no. A1301), CDK2 (cat.no. A2254), CyclinE (cat. no. A0112), p27 (cat. no. A0026) and $\beta$-actin (cat. no. AC006) were purchased from ABclonal (Boston, USA). Antibodies to detect total-Akt (cat. no. 4691T) and phosphorylated-Akt (p-Akt, Ser473, cat. no. 4060T) were purchased from Cell Signaling Technology (CST, Danvers, MA, USA). All of the antibodies mentioned above are rabbit anti-human antibodies and used in 1:1,000 dilutions except $\beta$-actin and p-Akt (1:2,000 dilution).

Cell viability and cell proliferation assay. Cytotoxicity was assessed by CCK-8 assay. Briefly, the indicated number of cells was seeded in 96-well plate and cultured for $24 \mathrm{~h}$. A series of concentrations of G-Rh2 $(30,40,50,60,70 \mu \mathrm{M})$ were used to treat glioma cells and an equal volume of DMSO (final concentration, $<0.1 \%$ ) was used as a control. After culturing for specified times, the cytotoxicity of G-Rh2 was measured using a CCK-8 according to the manufacturer's instructions. The absorbance was measured by Microplate reader (Thermo Fisher Scientific Inc.) at $450 \mathrm{~nm}$ wavelength.

For measuring cellular proliferation, clone formation assay was performed. Briefly, 150 A172 cells were cultured in 6-well plates for $24 \mathrm{~h}$ and followed by the treatment with different concentrations of G-Rh2 $(0,30,40,50,60 \mu \mathrm{M})$ for another 7 days. Cell clones were immobilized by methanol and stained with crystal violet Solution before photographed and counted. More than 50 cells were defined as a positive colony and the number of clones was counted with Image Pro Plus 6.0 (Media Cybernetics, Inc., Rockville, MD, USA). Colony formation rate $=($ number of clones/number of cells inoculated $) \times 100 \%$.

Flow cytometric analysis. Briefly, G-Rh2-treated cells were digested by EDTA-free Trypsin and collected by centrifugation at $400 \mathrm{~g}$ for $5 \mathrm{~min}$. Collected cells were washed once with Phosphate buffered saline (PBS). For cell cycle analysis, cells were fixed in $70 \%$ ethanol overnight at $4{ }^{\circ} \mathrm{C}$ and treated with RNaseA for $30 \mathrm{~min}$ in water bath, and resuspended in $400 \mu \mathrm{l}$ propidium iodide (PI) for $30 \mathrm{~min}$ in dark at $4^{\circ} \mathrm{C}$. For cell apoptosis analysis, collected cells were resuspended in $500 \mu \mathrm{l}$ binding buffer with $5 \mu \mathrm{l}$ AnnexinV-FITC and $5 \mu \mathrm{l} \mathrm{PI}$ for $15 \mathrm{~min}$ in dark at room temperature. Cell cycle distribution and apoptosis proportion was measured by flow cytometry (BD LSRFortessa ${ }^{\mathrm{TM}}$; BD Biosciences, San Diego, CA, USA). A total of $1.0 \times 10^{4}$ events were acquired for analysis using Cell Quest software.

Western blot assay. Proteins were extracted with Cell Total Protein Extraction Kit (KeyGEN BioTECH, Jiangsu, China). Briefly, G-Rh2 treated cells were lysed in RIPA lysis buffer (Contains Protease inhibitor, phosphatase inhibitors and PMSF) on ice for $30 \mathrm{~min}$. The supernatants were collected after centrifugation at $18.8 \times 10^{3} \mathrm{~g}$ at $4^{\circ} \mathrm{C}$ for $30 \mathrm{~min}$. Protein concentration was determined by Bradford method (KeyGEN BioTECH), and whole supernatants were mixed with 5xSDS loading buffer (contain 0.25M Tris-HCl, 1.6M MEDTA ( $\mathrm{pH}=8.5)$, DTT, LDS, glycerol, bromophenol blue, pyronin $\mathrm{Y}$ ) at a ratio of $4: 1$. Protein samples were heated at $100^{\circ} \mathrm{C}$ for $10 \mathrm{~min}$ and separated on SDS polyacrylamide gels. The Separating gel concentration was determined by the size of the target protein molecular weight. The separated proteins were then transferred to a PVDF membrane (Merck KGaA, Darmstadt, Germany), blocked with 5\% evaporated milk and probed with a primary antibody for $24 \mathrm{~h}$. After incubation with horseradish peroxidase (HRP)-conjugated second antibody (cat. no. bs-0295G; Bioss, Beijing, China), membrane blots were stained with enhanced chemiluminescent system (Fdbio Science, Hangzhou, China) to visualize the protein bands. Densitometry of western blots was performed with Quantity One v4.62 (Bio-Rad Laboratories, Richmond, CA, USA). The protein levels were first normalized to loading controls and then normalized to experimental controls. Images shown in the figures were representative from all individuals.

Statistical analysis. Data were analyzed by one-way analysis of variance (ANOVA) in SPSS19.0, followed by Dunnett-test when treated groups were compared only to the control group. For plotting the analysis findings software GraphPad Prism 6.0 was used and $\mathrm{P}<0.05$ was considered to indicate a statistically significant difference.

\section{Results}

G-Rh2 treatment decreases the viability of human glioma cells. Initially, for determining the effect of G-Rh2 on cell viability and Cytotoxicity, U87MG and A172 glioma cell lines were used. With the increased concentration of G-Rh2 and the prolongation of treatment period, the cell viability was decreased gradually (Fig. 1B and C). The IC50 of G-Rh2 exposure on A172 and U87MG cells for $24 \mathrm{~h}$ was 60.0 and $57.2 \mu \mathrm{M}$, respectively. Our findings demonstrated that G-Rh2 inhibits the viability of U87MG and A172 glioma cells in a dose/time-dependent manner. Especially, at $40 \mu \mathrm{M}$ and above concentrations or 12-48 $\mathrm{h}$ of incubation time, the treatment groups were statistically significant compared with the control group. 

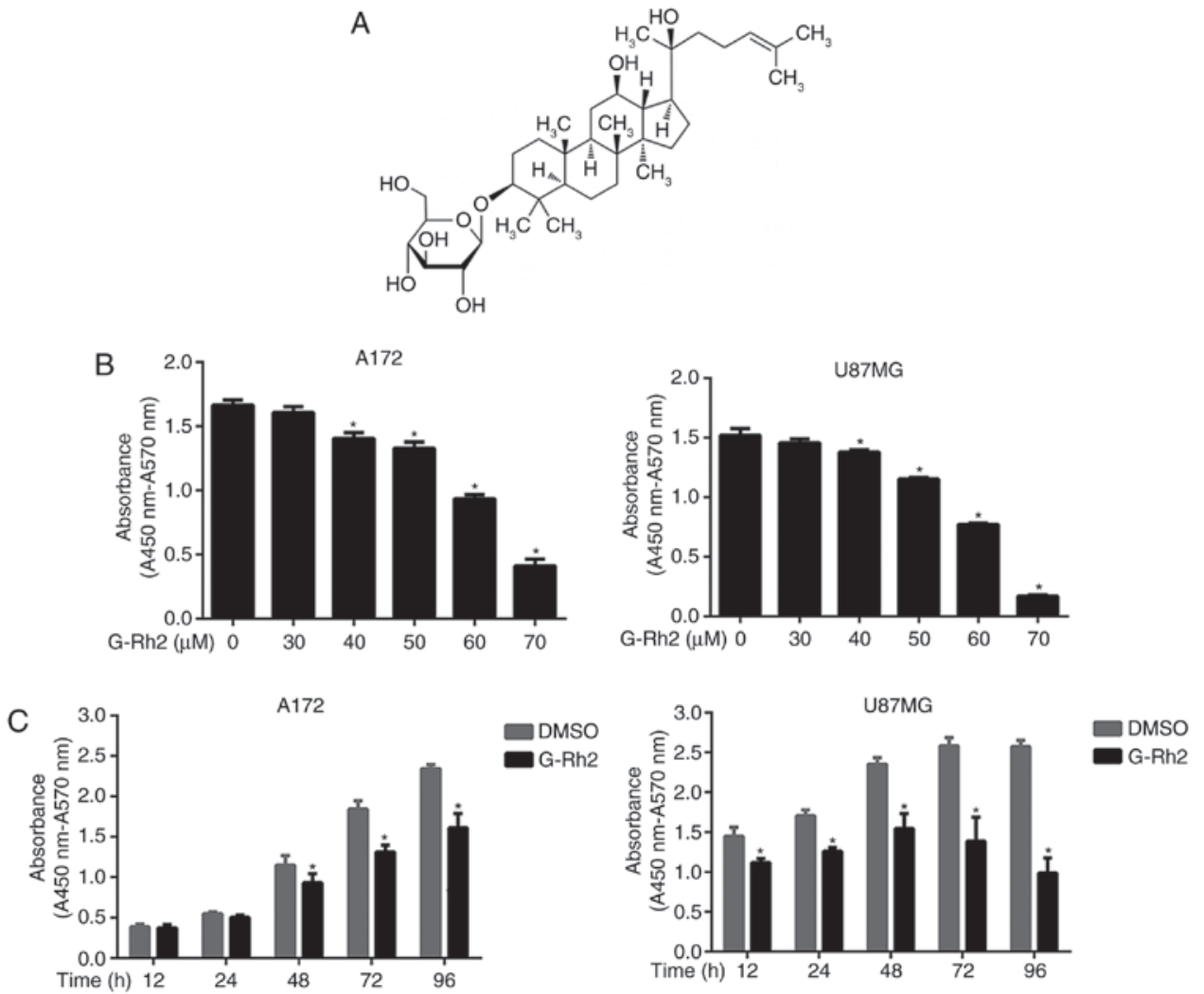

Figure 1. Cytotoxicity of G-Rh2 in human U87MG and A172 glioma cell lines. (A) Chemical structures of G-Rh2; (B) Exponentially growing U87MG and A172 $\left(5 \times 10^{3}\right.$ cells per well) were treated with series concentrations of G-Rh2 for $24 \mathrm{~h}$. With the increasing of G-Rh2 concentration, the viability decreased gradually in both cell lines, $0 \mu \mathrm{M}$ (DMSO) was used as a control; (C) U87MG $\left(5 \times 10^{3}\right.$ cells per well) and A172 (2x10 ${ }^{3}$ cells per well) were treated with G-Rh2 $(40 \mu \mathrm{M})$ for the indicated times. With the prolongation of treatment period, the viability decreased gradually in both cell lines. The data shown represent mean \pm SD of three independent experiments. "P<0.05 vs. corresponding control group.

G-Rh2 treatment decreases the clone formation of human Al72 glioma cells. We noticed that with G-Rh2 treatment glioma cells proliferation was decreased and the dead cells were increased in a dose-dependent manner (Fig. 2A). This demonstrates that G-Rh2 may have an impact on cell apoptosis and proliferation. We further performed clone formation assay to evaluate two important characters: Cell population dependence and proliferation ability. It can thus discriminate between cytotoxic (cell death) and cytostatic (decreased growth rate) effects (20). Our findings demonstrated that treatment with G-Rh2 significantly inhibited the clone formation of A172 cells in a dose-dependent manner (Fig. 2B and C).

$G$-Rh2 treatment induces the cell cycle arrest apoptosis in glioma cells. According clone formation assay, we speculated that G-Rh2 may exert an anti-tumor effect in A172 cells by inhibiting proliferation and inducing apoptosis. Therefore, we further analyzed by flow cytometry. We found that compared to untreated control group $(47.94 \pm 1.63 \%)$, the proportion of cells in G1 phase was significantly increased in the G-Rh2 treatment group $(51.48 \pm 7.05 \%$ at $30 \mu \mathrm{M}$ and $80.66 \pm 7.50 \%$ at $60 \mu \mathrm{M})$. Correspondingly, proportion of S-phase was markedly decreased $(37.07 \pm 8.50 \%$ at $30 \mu \mathrm{M}$ and $7.29 \pm 0.20 \%$ at $60 \mu \mathrm{M})$ compared with control group $(40.40 \pm 4.35 \%)$. Therefore, these results indicated that G-Rh2 was able to block the cell cycle progression and induce G1 phase arrest in A172 glioma cells (Fig. 3A). Moreover, our apoptotic analysis of A172 cells indicated that with G-Rh2 treatment apoptotic cells were increased

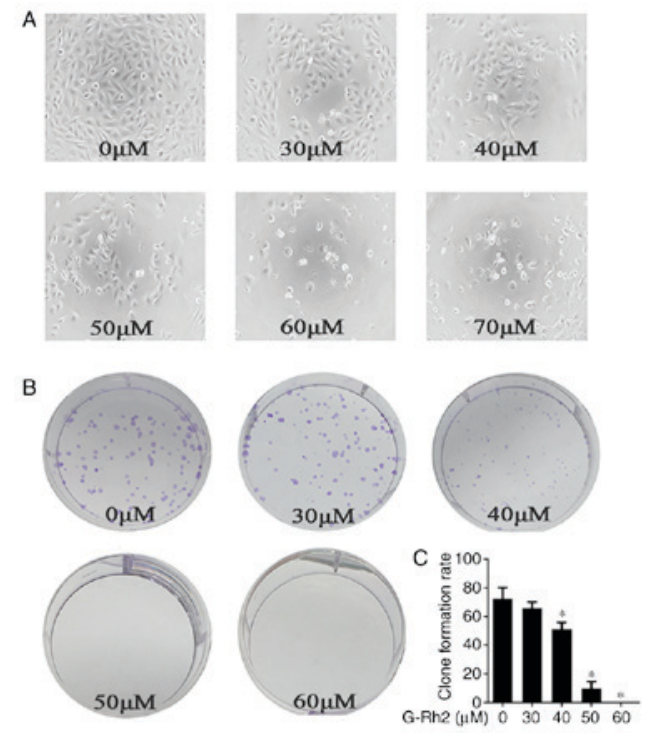

Figure 2. Effects of G-Rh2 on the proliferation in A172 glioma cells. (A) The A172 cells were seeded in 96 -well plates and were then treated with G-Rh2 at a series of concentration $(0,30,40,50,60,70 \mu \mathrm{M})$ for $36 \mathrm{~h}$. Morphological changes: Scattered and fragmented shapes, reduced cell density and increased cell gap were observed under Inverted optical microscope (magnification, x200); (B) The A172 cells were seeded at a density of 150 cells in 6-well plates and cultured for $48 \mathrm{~h}$ before treated with G-Rh2 at concentration $(0,30,40,50,60 \mu \mathrm{M})$, and then cultured for 7 days. With the G-Rh2 concentration increased, the number of clones decreased gradually. $0 \mu \mathrm{M}$ (DMSO) was used as a control and clone formation number were quantified. (C) The data shown represent mean $\pm \mathrm{SD}$ of three independent experiments. " $\mathrm{P}<0.05$ vs. corresponding control group. 

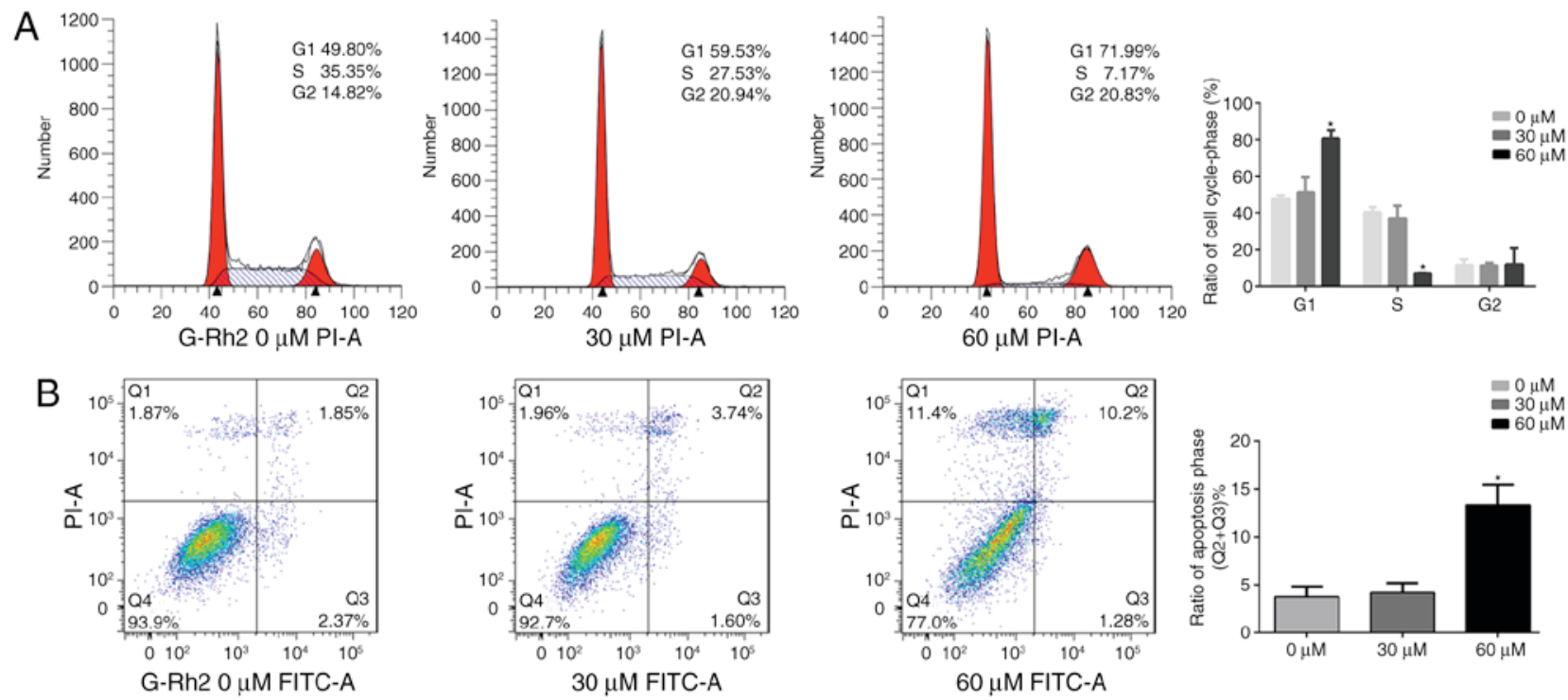

Figure 3. Effect of G-Rh2 on cell cycle and apoptosis. The A172 cells were treated with G-Rh2 at $0,30,60 \mu \mathrm{M}$ for $36 \mathrm{~h}$ and then flow cytometry analysis was performed according to the manufacturer's protocol. G-Rh2 exerts the inhibitory effect to block cell cycle in the G1 phase in a dose-dependent manner (A) and induce apoptosis. (B) $0 \mu \mathrm{M}$ (DMSO) was used as a control. The data shown represent mean $\pm \mathrm{SD}$ of three independent experiments. "P< $<0.05$ vs. corresponding control group.

significantly at $60 \mu \mathrm{M}(13.37 \%)$ compared to untreated control group (3.73\%) as showed in Fig. 3B.

G-Rh2 induces G1 phase arrest by inhibiting CDK4/CyclinD in A172 cells. Cell cycle related proteins are a class of proteins with specific periodicity, which can reflect the process of cell cycle (21). We found that upon treatment with a series of concentrations of G-Rh2, the CDK4 was decreased gradually (Fig. 4A and B). CyclinD, the periodic protein regulating CDK4 activity, was also decreased (Fig. 4A and B). We observed modestly decreased CDK2 protein expression and no obvious on CyclinE protein expression level with G-Rh2 treatment (Fig. 4C and D).We further investigated the $\mathrm{p} 27$ protein, one of the CDKIs family members, which play an important role in the regulation of G1-S transition to inhibit the activation of CDK2/CyclinE complex. Consistent with the CyclinE, there was no significant change after treatment with G-Rh2 in A172 cell line (Fig. 4C and E).

G-Rh2 reduces Akt expression and phosphorylation. Previous studies have shown that the phosphatidylinositol 3 kinase (PI3K)/Akt signaling pathway mediates cell proliferation, survival and motility. Akt is implicated in the oncogenesis of many cancers (22). Therefore, we examined the effect of $\mathrm{G}-\mathrm{Rh} 2$ treatment on Akt expression and phosphorylation. The expression of total-Akt protein was decreased gradually and p-Akt (Ser473) also affected by the treatment with G-Rh2. Therefore, the results suggested that G-Rh2 might be exerting its anti-proliferative effects by inhibiting Akt expression and phosphorylation, at least partially (Fig. 5A and B).

\section{Discussion}

Glioma patients benefit little from standard therapy (Surgical resection combined with radiotherapy and chemotherapy). How to improve bioavailability and penetrate the BBB have been obstacles in chemotherapy drugs. Animal experiments show that the bioavailability of G-Rh2 is about $16 \%$ and it distributed mainly to the liver and gastrointestinal tissues. After oral administration at $3 \mathrm{mg} / \mathrm{kg}$, the concentration in plasma was $0.104 \mu \mathrm{M}$ in dogs (4). Our findings demonstrated that G-Rh2 inhibits the viability of U87MG and A172 glioma cells in a dose/time-dependent manner. The IC50 of G-Rh2 exposure on A172 and U87MG for $24 \mathrm{~h}$ was 60.0 and $57.2 \mu \mathrm{M}$, respectively. It is necessary to develop new methods of administration or to change the physical and chemical properties so that it can pass through BBB. Recent studies have provided us some new ideas. For example, liposomes, the small spherical vesicle with single or multiple lipid bilayers, have been widely exploited due to their unique characteristics. Animal experiments showed that the concentration of docetaxel from docetaxel-loaded nanoliposomes in the brain was significantly increased (23). Esterification of G-Rh2 enhanced its cellular uptake and antitumor activity in Human HepG2 Cells (24). The combination of G-Rh2 with magnetic iron oxide nanoparticles increased the cell uptake efficiency and convection-enhanced delivery (CED) treatment may break through the BBB and greatly increased the concentration of drugs in the brain. But their safety needs further evaluation (25).

$\mathrm{G}-\mathrm{Rh} 2$ is classified as a new anticancer drug owing to its capability of inducing apoptosis in multiple tumor cells (9-11). In recent years, another utility of G-Rh2 in inhibiting tumor cell proliferation has been noticed. Previous studies suggests that Rh2-mediated cell cycle arrest in MCF-7 cells was accompanied by the down-regulation of CDK and Cyclins, reduced interaction between CyclinD and CDK4/CDK6 and increased recruitment of p15INK4B and p27KIP1 to CDK4/CyclinD and CDK6/CyclinD complexes (26). In addition, the Rh2-mediated G0/G1 phase cell cycle arrest in A549 lung adenocarcinoma cells has been reported to be correlated with reduced expression of CDK6, CyclinD1 and CyclinE, but levels of CDK4, CDK2 and CyclinA were unaltered (27). These researches 
A

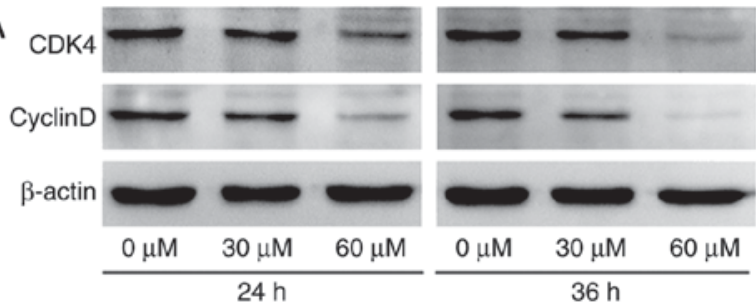

C

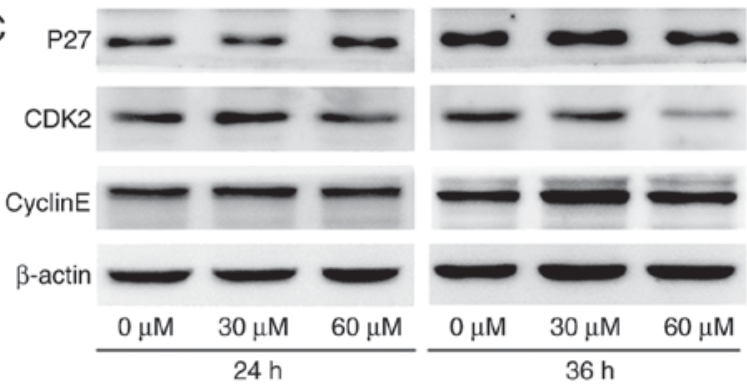

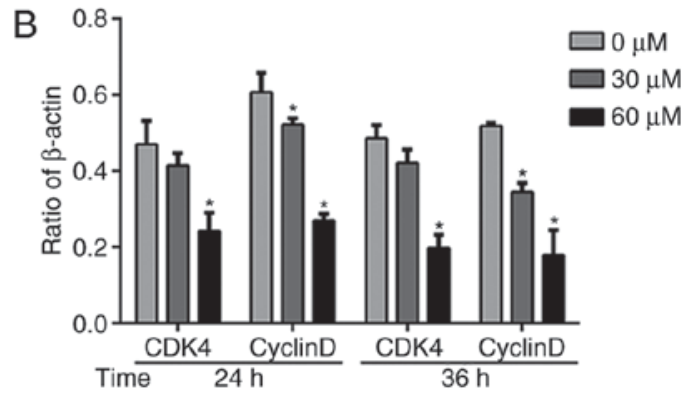

D

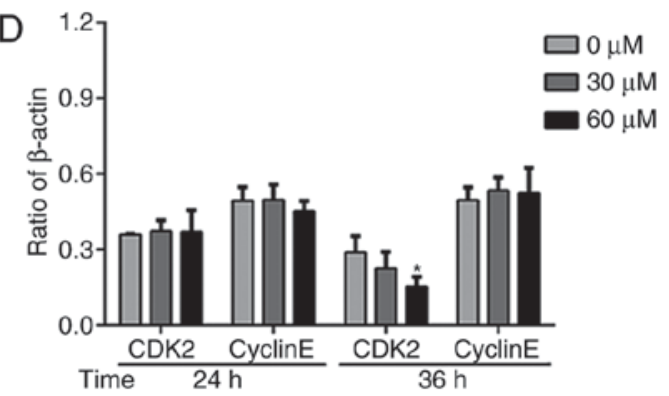

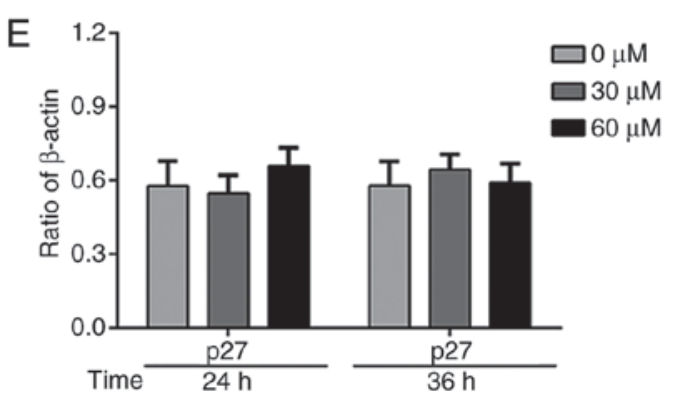

Figure 4. G-Rh2 inhibits cell cycle related protein CDK4/Cyclin D in A172 cells. The A172 cells were treated with G-Rh2 at $0,30,60 \mu \mathrm{M}$ for 24 or $36 \mathrm{~h}$, and then western blotting was performed for protein analysis. (A and B) the CDK4 and Cyclin D protein decreased gradually with a dose-dependent manner. (C to E) CDK2 protein expression modestly decreased but no obvious difference observed on CyclinE and p27 protein level with G-Rh2 treatment. $0 \mu \mathrm{M}$ (DMSO) was used as a control. The protein level were quantified by scanning densitometry and normalized to $\beta$-actin (as an intracellular control). The data shown represent mean \pm SD of three independent experiments. ${ }^{*} \mathrm{P}<0.05$ vs. corresponding control group.
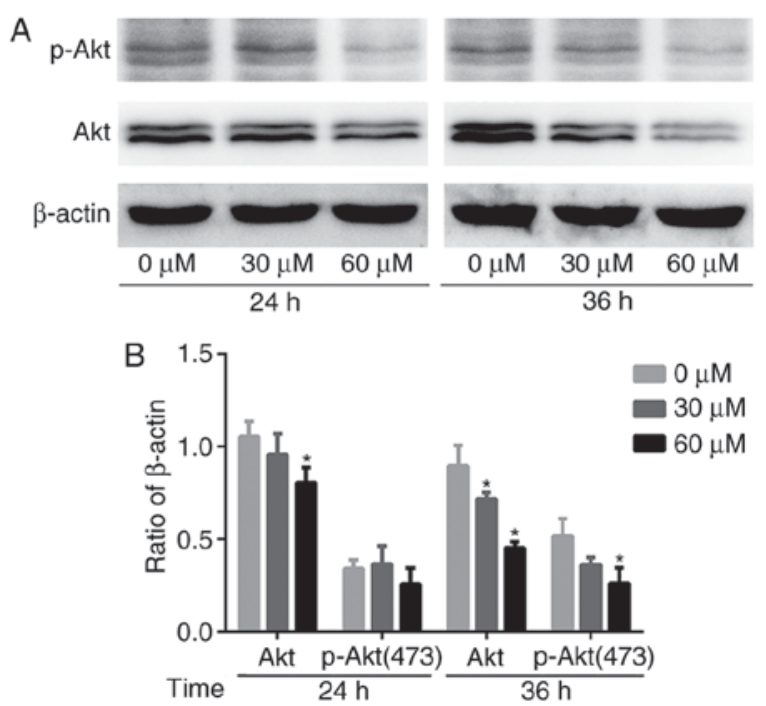

Figure 5. G-Rh2 exerts anti-proliferation effect by inhibiting Akt expression and phosphorylation. The A172 cells were treated with G-Rh2 at $0,30,60 \mu \mathrm{M}$ for 24 or $36 \mathrm{~h}$, and followed protein analysis. (A) The protein expression level of total Akt and p-Akt (Ser473) decreased gradually with the increase of G-Rh2 concentration and (B) protein expression level were quantified by scanning densitometry and normalized to $\beta$-actin (as an intracellular control). The data shown represent mean $\pm \mathrm{SD}$ of three independent experiments. ${ }^{*} \mathrm{P}<0.05$ vs. corresponding control group. indicated the anti-tumor mechanisms involved may be cell line specific.

A172 and U87MG are classic glioma cell lines and are derived from glioblastoma. Considering the heterogeneity of cell response to G-Rh2, the cytotoxicity of G-Rh2 was tested at the two cell lines. The results showed the reactivity to G-Rh2 was consistent on both cell lines. Previous studies had shown that G-Rh2 inhibited the expression of EGFR in A172 (19), thus we continued to explore the following mechanism in the A172. Our further investigation revealed that cells were arrested at G1 phase of cell cycle and the expression of cell cycle related proteins CDK4/CyclinD was decreased gradually with G-Rh2 treatment, which suggest that CDK4/CyclinD is an essential regulatory target of G-Rh2 for blocking cell cycle progression. As cell cycle progresses, CyclinE expression is gradually increased in early G1 phase and in G1 to S transition CDK2/CyclinE complex exerts a dominant kinase activity. We found that with G-Rh2 treatment CyclinE level was unaltered, whereas CDK2 expression is modestly reduced. Further investigation revealed that $\mathrm{p} 27$, the main inhibitor of $\mathrm{CDK} 2 /$ CyclinE, was unaltered as well. These results further suggested that G-Rh2 blocked cells cycle in the G1 phase. Previous studies indicated that G-Rh2 only inhibited the kinase activity of CDKs rather than protein expression (18). 
Whether G-Rh2 exerts inhibition of CDKs activity in such manner in glioma cells needed to be explored.

EGFR mutations are detected in half of gliomas, which may contribute to tumorgenesis (28). The research indicated G-Rh2 may have its anti-tumor effect through inhibiting EGFR expression in A172 glioma cells (19). But the downstream molecules have not yet been elucidated. The serine/threonine kinase $A k t$, also known as protein kinase $B(P K B)$, is one of the downstream signaling molecules regulated by EGFR. Since its discovery as a proto-oncogene, Akt has become a major focus of attention because of its critical regulatory role in diverse cellular processes, including cancer progression $(29,30)$. So we decided to investigate the impact of G-Rh2 on Akt. Our results showed that with G-Rh2 treatment, total-Akt levels were decreased, and the Akt phosphorylation was inhibited in a dose-dependent manner. This finding indicates that G-Rh2 could exert anti-tumor activity through inhibiting Akt pathway in glioma cells. Abnormal Akt activation was observed in the majority of GBM (31). Akt phosphorylation inhibitors were able to block effectively the proliferation, migration and invasion in glioma cells (32). These studies suggest that inhibition of Akt pathway is an effective strategy to target glioma. Dozens of Akt inhibitors being in the Phase II/III clinical trials, but so far they have not really been used in clinical patients. Some key points such as differences in pharmacokinetic properties and high toxicity have not been fully resolved (33). Drug combination therapy is well worth promoting for which contributes to enhance the curative effect and reduce drug resistance. PI3K/Akt inhibitors have been evaluated for their function to resensitize glioma cells to TMZ (22). Our findings suggest that G-Rh2 may serve as a potential Akt inhibitor and provide a new alternative for the development of glioma treatment. Furthermore, G-Rh2 is reported to suppress the growth of $\mathrm{H} 22$ hepatomas without causing severe side effects in mouse model, which might be a reference for safe drug use (34). But the superior advantages compared to Akt inhibitor need further study.

In summary, our study demonstrates that G-Rh2 exerts an anti-tumor effect on glioma cells via mechanisms that modulate the expression of CDK4/CyclinD complex and Akt, might have potential applications as an effective anti-cancer drug for glioma.

\section{Acknowledgements}

The present study was supported by the Guangdong Natural Science Foundation (2016A030313525); the Science and Technology Program of Guangzhou (201607010015); the Guangdong Province Medical Research Foundation (A2015381) and the President Foundation of Nanfang Hospital, Southern Medical University (2014C016). We would like to thank Professor Qinghe Meng for the suggestion and guidance.

\section{References}

1. Louis DN, Ohgaki H, Wiestler OD, Cavenee WK, Burger PC, Jouvet A, Scheithauer BW and Kleihues P: The 2007 WHO classification of tumours of the central nervous system. Acta Neuropathol 114: 97-109, 2007.

2. Ostrom QT, Bauchet L, Davis FG, Deltour I, Fisher JL, Langer CE, Pekmezci M, Schwartzbaum JA, Turner MC, Walsh KM, et al: The epidemiology of glioma in adults: A 'state of the science' review. Neuro-Oncol 16: 896-913, 2014
3. Wang H, Xu T, Jiang Y, Xu H, Yan Y, Fu D and Chen J: The challenges and the promise of molecular targeted therapy in malignant gliomas. Neoplasia 17: 239-255, 2015.

4. Gu Y, Wang GJ, Sun JG, Jia YW, Wang W, Xu MJ, Lv T, Zheng YT and Sai Y: Pharmacokinetic characterization of ginsenoside Rh2, an anticancer nutrient from ginseng, in rats and dogs. Food Chem Toxicol 47: 2257-2268, 2009.

5. Bahrke MS and Morgan WR: Evaluation of the ergogenic properties of ginseng: An update. Sports Med 29: 113-133, 2000.

6. Jin Y, Kotakadi VS, Ying L, Hofseth AB, Cui X, Wood PA, Windust A, Matesic LE, Pena EA, Chiuzan C, et al: American ginseng suppresses inflammation and DNA damage associated with mouse colitis. Carcinogenesis 29: 2351-2359, 2008.

7. Shen CY, Jiang JG, Yang L, Wang DW and Zhu W: Anti-ageing active ingredients from herbs and nutraceuticals used in traditional Chinese medicine: Pharmacological mechanisms and implications for drug discovery. Br J Pharmacol 174: 1395-1425, 2017.

8. Cui X, Jin Y, Poudyal D, Chumanevich AA, Davis T, Windust A, Hofseth A, Wu W, Habiger J, Pena E, et al: Mechanistic insight into the ability of American ginseng to suppress colon cancer associated with colitis. Carcinogenesis 31: 1734-1741, 2010.

9. Li B, Zhao J, Wang CZ, Searle J, He TC, Yuan CS and Du W: Ginsenoside Rh2 induces apoptosis and paraptosis-like cell death in colorectal cancer cells through activation of p53. Cancer Lett 301: 185-192, 2011.

10. Park HM, Kim SJ, Kim JS and Kang HS: Reactive oxygen species mediated ginsenoside $\mathrm{Rg} 3$ - and $\mathrm{Rh} 2$-induced apoptosis in hepatoma cells through mitochondrial signaling pathways. Food Chem Toxicol 50: 2736-2741, 2012.

11. Guo XX, Li Y, Sun C, Jiang D, Lin YJ, Jin FX, Lee SK and Jin YH: p53-dependent Fas expression is critical for Ginsenoside $\mathrm{Rh} 2$ triggered caspase-8 activation in HeLa cells. Protein Cell 5: 224-234, 2014.

12. Park EK, Lee EJ, Lee SH, Koo KH, Sung JY, Hwang EH, Park JH, Kim CW, Jeong KC, Park BK and Kim YN: Induction of apoptosis by the ginsenoside $\mathrm{Rh} 2$ by internalization of lipid rafts and caveolae and inactivation of Akt. Br J Pharmacol 160: 1212-1223, 2010

13. Liu S, Chen M, Li P, Wu Y, Chang C, Qiu Y, Cao L, Liu Z and Jia C: Ginsenoside rh 2 inhibits cancer stem-like cells in skin squamous cell carcinoma. Cell Physiol Biochem 36: 499-508, 2015.

14. Hengstschläger M, Braun K, Soucek T, Miloloza A and Hengstschläger-Ottnad E: Cyclin-dependent kinases at the G1-S transition of the mammalian cell cycle. Mutat Res 436: 1-9, 1999.

15. Giacinti $\mathrm{C}$ and Giordano A: RB and cell cycle progression. Oncogene 25: 5220-5227, 2006.

16. Munro S, Carr SM and La Thangue NB: Diversity within the $\mathrm{pRb}$ pathway: Is there a code of conduct. Oncogene 31: 4343-4352, 2012.

17. Lapenna S and Giordano A: Cell cycle kinases as therapeutic targets for cancer. Nat Rev Drug Discov 8: 547-566, 2009.

18. Chung KS, Cho SH, Shin JS, Kim DH, Choi JH, Choi SY, Rhee YK, Hong HD and Lee KT: Ginsenoside Rh2 induces cell cycle arrest and differentiation in human leukemia cells by upregulating TGF- $\beta$ expression. Carcinogenesis 34: 331-340, 2013.

19. Li S, Gao Y, Ma W, Guo W, Zhou G, Cheng T and Liu Y: EGFR signaling-dependent inhibition of glioblastoma growth by ginsenoside Rh2. Tumor Biol 35: 5593-5598, 2014.

20. Plumb JA: Cell sensitivity assays: Clonogenic assay. Methods Mol Med 88: 159-164, 2004.

21. Diaz-Moralli S, Tarrado-Castellarnau M, Miranda A and Cascante M: Targeting cell cycle regulation in cancer therapy. Pharmacol Ther 138: 255-271, 2013.

22. Burris HA III: Overcoming acquired resistance to anticancer therapy: Focus on the PI3K/AKT/mTOR pathway. Cancer Chemother Pharmacol 71: 829-842, 2013.

23. Shaw TK, Mandal D, Dey G, Pal MM, Paul P, Chakraborty S, Ali KA, Mukherjee B, Bandyopadhyay AK and Mandal M: Successful delivery of docetaxel to rat brain using experimentally developed nanoliposome: A treatment strategy for brain tumor. Drug Deliv 24: 346-357, 2017.

24. Chen F, Deng ZY, Zhang B, Xiong ZX, Zheng SL, Tan CL and $\mathrm{Hu}$ JN: Esterification of Ginsenoside Rh2 enhanced its cellular uptake and antitumor activity in human HepG2 cells. J Agric Food Chem 64: 253-261, 2016.

25. Kaluzova M, Bouras A, Machaidze R and Hadjipanayis CG: Targeted therapy of glioblastoma stem-like cells and tumor non-stem cells using cetuximab-conjugated iron-oxide nanoparticles. Oncotarget 6: 8788-8806, 2015. 
26. Choi S, Kim TW and Singh SV: Ginsenoside Rh2-mediated G1 phase cell cycle arrest in human breast cancer cells is caused by p15Ink4B and p27Kip1-dependent inhibition of cyclin-dependent kinases. Pharm Res 26: 2280-2288, 2009.

27. Cheng CC, Yang SM, Huang CY, Chen JC, Chang WM and Hsu SL: Molecular mechanisms of ginsenoside Rh2-mediated G1 growth arrest and apoptosis in human lung adenocarcinoma A549 cells. Cancer Chemother Pharmacol 55: 531-540, 2005.

28. Viana-Pereira M, Lopes JM, Little S, Milanezi F, Basto D, Pardal F, Jones C and Reis RM: Analysis of EGFR overexpression, EGFR gene amplification and the EGFRvIII mutation in portuguese high-grade gliomas. Anticancer Res 28: 913-920, 2008.

29. Hill MM and Hemmings BA: Inhibition of protein kinase B/Akt: Implications for cancer therapy. Pharmacol Ther 93: 243-251, 2002.

30. Yoeli-Lerner $\mathrm{M}$ and Toker A: Akt/PKB signaling in cancer: $\mathrm{A}$ function in cell motility and invasion. Cell Cycle 5: 603-605, 2006.
31. Gallia GL, Tyler BM, Hann CL, Siu IM, Giranda VL, Vescovi AL, Brem H and Riggins GJ: Inhibition of Akt inhibits growth of glioblastoma and glioblastoma stem-like cells. Mol Cancer Ther 8: 386-393, 2009.

32. Chautard E, Ouédraogo ZG, Biau J and Verrelle P: Role of Akt in human malignant glioma: From oncogenesis to tumor aggressiveness. J Neurooncol 117: 205-215, 2014.

33. Mayer IA and Arteaga CL: The PI3K/AKT pathway as a target for cancer treatment. Annu Rev Med 67: 11-28, 2016.

34. Lv Q, Rong N, Liu LJ, Xu XL, Liu JT, Jin FX and Wang CM: Antitumoral activity of (20R)- and (20S)-Ginsenoside Rh2 on transplanted hepatocellular carcinoma in mice. Planta Med 82: 705-711, 2016.

This work is licensed under a Creative Commons Attribution-NonCommercial-NoDerivatives 4.0 International (CC BY-NC-ND 4.0) License. 